\title{
Measurement of the Forward-Backward Asymmetry in the Drell-Yan Dilepton Production in Proton-Proton Collisions at the CMS Experiment at the LHC
}

\author{
V. Shalaev ${ }^{1, \star}$, I. Gorbunov ${ }^{1, \star \star}$, and S. Shmatov ${ }^{1, \star \star \star}$ \\ ${ }^{1}$ JINR, Dubna, Russia
}

\begin{abstract}
In this paper we review the results of a measurement of the forward-backward asymmetry of oppositely charged lepton pairs produced via $Z / \gamma^{\star}$ boson in pp collisions during the LHC Run 1 at $\sqrt{s}=8 \mathrm{TeV}$ with integrated luminosity $19.1 \mathrm{fb}^{-1}$ (2012). We also present our preliminary results obtained with Monte Carlo samples at $\sqrt{s}=13 \mathrm{TeV}$.
\end{abstract}

\section{Introduction}

The study of the lepton pairs production in the Drell-Yan processes [1], $q \bar{q} \rightarrow \gamma^{*} / Z \rightarrow l^{+} l^{-}$(Fig. 1a), is of extreme importance: these processes allow us to verify the Standard Model (SM) predictions for the elementary particles interaction at the new energy region and to search for new physics beyond the SM $[2,3]$. The presence in the Drell-Yan processes both the vector $\left(g_{V}\right)$ and axial-vector $\left(g_{A}\right)$ current results in the appearance of an asymmetry in the lepton angular distributions due to $P$-parity violation:

$$
\frac{d \sigma}{d\left(\cos \theta^{*}\right)}=\frac{4 \pi \alpha^{2}}{3 s}\left[\frac{3}{8} A\left(1+\cos ^{2} \theta^{\star}\right)+B \cos \theta^{\star}\right]
$$

where $\theta^{\star}$ is the angle of lepton emission with respect to the direction of the quark momentum in the center mass of the lepton pair system (Fig. 1b), $A$ and $B$ - the coefficients dependent on weak isospin and a charge of fermions in the initial states. In general the value of the asymmetry given by the coefficient $B$ depends on the values of vector $\left(g_{V}\right)$ and axial-vector $\left(g_{A}\right)$ couplings which makes $B$ sensitive to the presence of additional contributions to the SM processes. Therefore, the possible difference between the asymmetry value and the SM predictions can indicate the existence of processes beyond the SM namely new neutral gauge bosons [4], the internal structure of quarks and leptons [5], supersymmetric particles and extra dimension [6]. Moreover measurement of the exact value of the asymmetry could shed light on the new phenomena nature such as the origin of a new particle and which theoretical model predicts the observed effect. For example, one of the distinguishing features of the asymmetry is its dependence on the pseudorapidity of the new neutral gauge boson predicted by various models with extended gauge sector [7]. This will allow us to choose between different

\footnotetext{
^e-mail: Vladislav.Shalaev@cern.ch

$\star \star$ e-mail: Ilya.Gorbunov@cern.ch

$\star \star \star$ e-mail: Sergei.Shmatov@cern.ch
} 
theoretical scenarios even for particles having the same spin. Also, a measurement of the asymmetry value may provide information indicative of the correctness of our understanding of the quark and gluons distribution functions. Moreover, the measurement of the asymmetry in the $Z$-pole allows us to measure the effective weak mixing angle $\sin ^{2} \theta_{W}$ values [9].

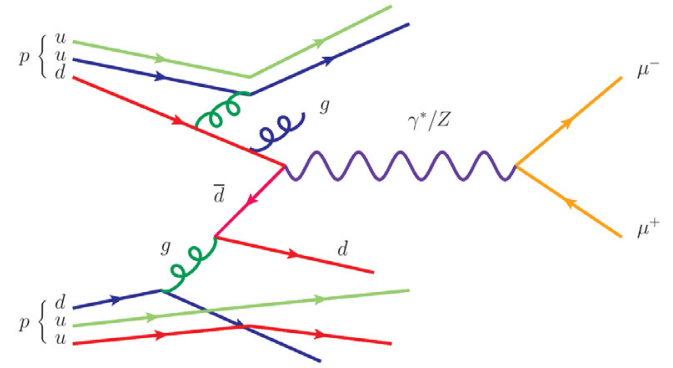

(a)

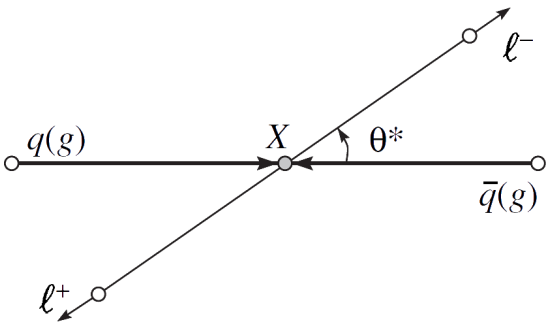

(b)

Figure 1. (a) Dilepton production in the annihilation of a quark-antiquark pair, $q \bar{q} \rightarrow \gamma^{*} / Z \rightarrow l^{+} l^{-}$. Definition of the angle $\theta^{*}$ in the decay of the exchanged particle X.

In this paper we review the results of a measurement of the forward-backward asymmetry of oppositely charged lepton pairs (both $\mu^{+} \mu^{-}$and $e^{+} e^{-}$) in pp collisions during the LHC Run 1 at $\sqrt{s}=$ $8 \mathrm{TeV}$ [10]. The data sample corresponds to an integrated luminosity of $19.7 \mathrm{fb}^{-1}$ collected by the CMS detector(2012). We also present our preliminary results obtained with Monte Carlo samples at $\sqrt{s}=13 \mathrm{TeV}$.

\section{CMS Experiment}

The central part of the experimental setup [8] is superconductive magnet solenoid. It is $13 \mathrm{~m}$ long and $6 \mathrm{~m}$ internal diameter, providing magnetic field of $3.8 \mathrm{~T}$. The magnetic system contains a tracker and calorimetric systems. The tracker system covers the pseudorapidity range $|\eta| \leqslant 2.5$ and includes two types of silicon detectors: a pixel detector and a silicon strip detector. The pixel detector is located near by the beam interaction point and consists of 65 million Individual sensitive elements namely pixel size of $100 \times 150 \mu \mathrm{m}$ grouped by in three layers with radii of 4,7 and $11 \mathrm{~cm}$. The strip detector is assembled from 10 million silicon strips which are located at a distance up to $130 \mathrm{~cm}$ from the beam axis.

The solenoid is surrounded by detector consisted of three types of muon system such as drift tubes in the central part of the CMS $(|\eta| \leqslant 1.2)$, cathode strip chambers in the front part $(1.9 \leqslant|\eta| \leqslant 2.4)$ and the resistive plates chambers in the entire range of pseudorapidity of a muon system $(|\eta| \leqslant 2.4)$. Joint use of the muon system and the tracker system allows us to measure the transverse muon momentum with a resolution between 1 and 5\% for values of transverse momentum up to $1 \mathrm{TeV}$.

\section{$3 A_{F B}$ measurements with $8 \mathrm{TeV}$ Data}

In the experiment the asymmetry can be obtained by means of an independent measurements of the number of dilepton events for the different invariant mass bins in the forward and backward regions defined by the polar angle $\theta$ of the lepton with respect to the quark momentum direction:

$$
A_{F B}=\frac{\sigma_{F}-\sigma_{B}}{\sigma_{F}+\sigma_{B}}, \quad \sigma_{F}=\int_{0}^{1} \frac{d \sigma}{d\left(\cos \theta^{\star}\right)} d\left(\cos \theta^{\star}\right), \quad \sigma_{B}=\int_{-1}^{0} \frac{d \sigma}{d\left(\cos \theta^{\star}\right)} d\left(\cos \theta^{\star}\right),
$$


where $\sigma_{F}$ and $\sigma_{B}$ are the total cross section for the events in the forward and backward directions. The direction "forward" ("backward") is given by a hemisphere with $\cos \theta^{\star} \geqslant(<0)$, where $\theta$ is the angle between the outgoing lepton $l^{-}$and quark $q$ in the lepton pair rest system. This definition suggests that the initial direction of the quark is known, but this condition is not valid in the case of experiments on pp-collisions. However, it is possible to fix the direction of the quark momentum by the two-lepton system emission direction since the pair is produced as a result of a predominantly valence quark and a sea antiquark annihilation and the momentum of the valence quark is on average larger than the momentum of the sea antiquark. The asymmetry defined this way allow us to study the spin structure of a muon pair produced in symmetric proton beams. We note that this approximation causes an increase in systematic uncertainties due to the errors in quark direction determination that must be taken into account while calculating asymmetry values.

To reduce uncertainties due to a nonzero transverse momentum of the quarks the Collins-Soper [11] coordinate system was used. In this system $\theta_{C S}^{\star}$ is the angle between the muon momentum and the $\mathrm{z}$-axis which halves the angle between the quark and antiquark momentum. The $A_{F B}$ was measured by analyzing the leptons angular distributions for each invariant mass bin. By using quantities written in the laboratory frame, one can represent the angle $\theta_{C S}^{\star}$ in the form

$$
\cos \theta_{C S}^{\star}=\frac{2\left(P_{1}^{+} P_{2}^{-}-P_{1}^{-} P_{2}^{+}\right)}{\sqrt{Q^{2}\left(Q^{2}+Q_{T}^{2}\right)}},
$$

where $Q$ and $Q_{T}$ are 4-momenta and transverse momentum of dilepton system, respectively; $P_{1,2}^{i}$ - the $i$ component of lepton and antilepton 4-momenta; and $P_{i}^{ \pm}=2^{-1 / 2}\left(P_{i}^{0} \pm P_{i}^{3}\right)$. In this case, the direction "forward" ("backward") is determined by the cosine sign: $\cos \theta_{C S}^{\star} \geqslant 0(<0)$.

The $\cos \theta_{C S}^{\star}$ distributions for $\mu \mu$ events at $\sqrt{s}=8 \mathrm{TeV}$ are presented in the Fig. 2. Only statistical uncertainties are shown. The stacked histograms represent the sum of the background contribution and the signal. The plots on the left (right) panels correspond to events with dilepton invariant mass 50 $<m<60 \mathrm{GeV}(133<m<150 \mathrm{GeV})$. The Monte Carlo predictions are normalized to the integrated luminosity of the data.
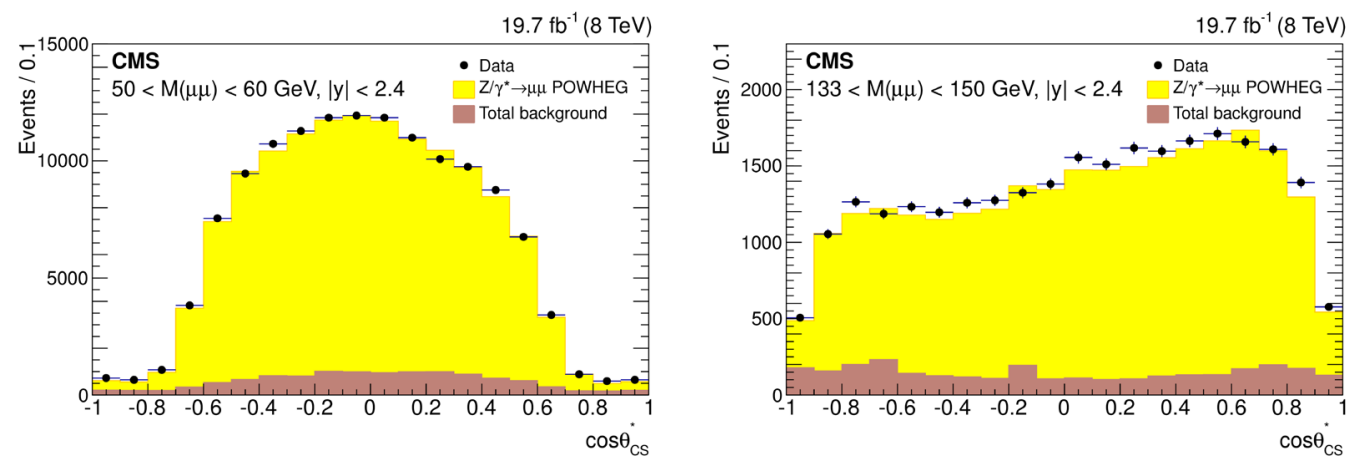

Figure 2. The $\cos ^{\star} \theta_{C S}$ distributions for $\mu \mu$ events at $\sqrt{s}=8 \mathrm{TeV}[10]$.

The measurement of $A_{F B}$ is performed for dilepton masses between $40 \mathrm{GeV}$ and $2 \mathrm{TeV}$ in five regions of absolute rapidity: $0-1,1-1.25,1.25-1.5,1.5-2.4$, and 2.4-5. The Fig. 3 shows the combined $\left(\mu^{+} \mu^{-}\right.$and $\left.e^{+} e^{-}\right)$unfolded $A_{F B}$ distributions in the four central rapidity regions. The statistical 
(thick vertical bar) and statistical plus systematics (thin vertical bar) uncertainties are presented. The measurements are compared with the next-leading-order prediction of Standard Model (POWHEG Monte Carlo generator [12]). The total uncertainties (considering the statistical, PDF, and scale uncertainties) in the powheg prediction are shown as shaded bands. The lower panel in each plot shows the difference of $A_{F B}$ in data and prediction divided by the total uncertainty of data and prediction.
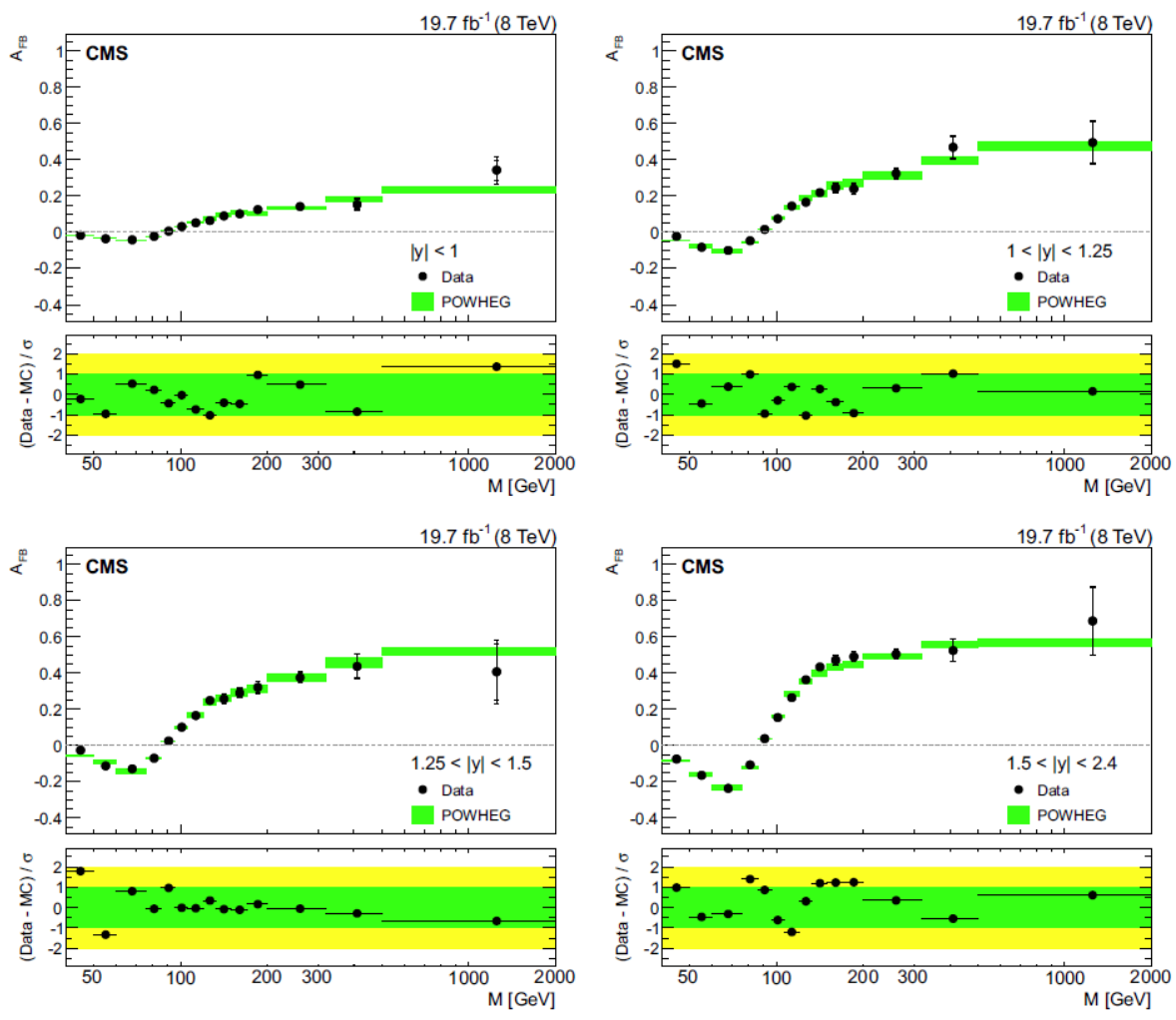

Figure 3. $A_{F B}$ distributions for different invariant mass and rapidity at $\sqrt{s}=8 \mathrm{TeV}[10]$.

\section{Monte Carlo Studies at $13 \mathrm{TeV}$}

The LHC operation at $\sqrt{s}=13 \mathrm{TeV}$, referred to as the LHC Run 2, started in 2015, and by the end of 2017 year the integrated luminosity collected by the CMS experiment reached 37.8 and $45.1 \mathrm{fb}^{-1}$ for 2016 and 2017, respectively.

The first study of the forward-backward asymmetry $A_{F B}$ at $\sqrt{s}=13 \mathrm{TeV}$ was performed with the Monte Carlo data. To compare data collected during the Run 2 with the SM predictions, a simulation of both signal and background events with the PYTHIA 8.0 Monte Carlo events generator was completed [13].

For example, the $\cos \theta_{C S}^{\star}$ distribution of dimuon events is given at Fig. 4a. Fig.4b shows the dependence of the simulated asymmetry $A_{F B}$ on the dimuon invariant mass. Presented distributions were obtained with the Monte Carlo generator without simulation of the particle response in the CMS detecting system. 
A simulation of events signals obtained with PYTHIA 8 and passed to a full Geant 4 simulation of the particle response in the CMS detecting systems, with using vital selection criteria and event reconstruction algorithms was performed but this results are not presented in this paper.

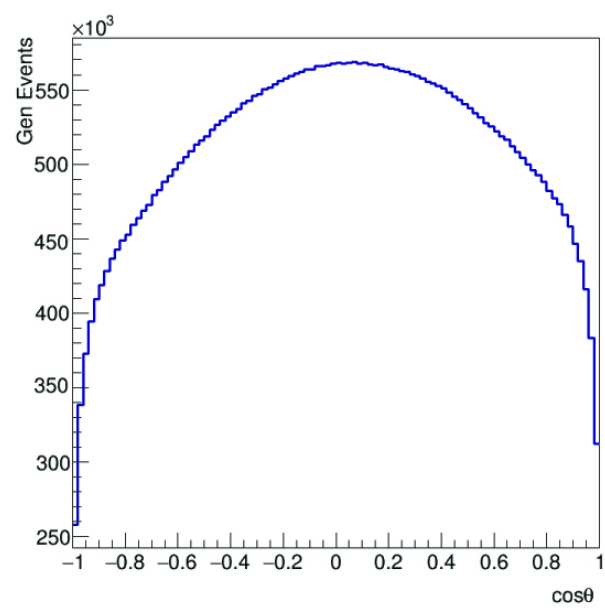

(a)

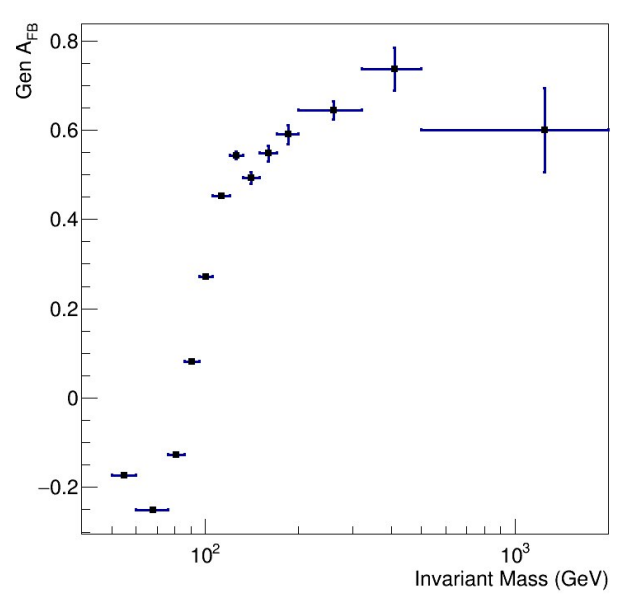

(b)

Figure 4. The results of simulation within the CMS at $\sqrt{s}=13 \mathrm{TeV}$ (without detector response): (a) the $\cos \theta_{C S}^{\star}$ distribution of dimuon events; (b) $A_{F B}$ distribution for different dimuon invariant mass.

We would like to thank the management of the CMS theme at JINR for support.

\section{References}

[1] S. D. Drell and T.-M. Yan, Phys. Rev. Lett. 25, 316 (1970).

[2] CMS Collab. (G. L. Bayatian et al.), J. Phys. G 34, 995 (2007).

[3] V. A. Rubakov, Phys. Usp. 55, 949 (2012); A. V. Lanev, Phys. Usp. 57, 923 (2014); Phys. Atom. Nucl. 78, 521 (2015); M. V. Savina, Phys. Atom. Nucl. 78, 532 (2015); S. V. Shmatov, Phys. Atom. Nucl. 76, 1106 (2013); Phys. Atom. Nucl. 78, 509 (2015); Phys. Atom. Nucl. 79, 266 (2016); in Proc. Joint RDMS CMS Seminar on Physics at LHC (2016), vol. 4, p. 13.

[4] D. London and J. L. Rosner, Phys. Rev. D 34, 1530 (1986); J. L. Rosner, Phys. Rev. D 35, 2244 (1987); Phys. Rev. D 54, 1078 (1996); A. Bodek and U. Baur, hep-ph/0102160; F. Abe et al., Phys. Rev. Lett. 79,2192 (1997).

[5] F. Abe et al., Phys. Rev. Lett. 79, 2198 (1997).

[6] H. Davoudiasl, J. L. Hewett, and T. G. Rizzo, Phys. Rev. Lett. 84, 2080 (2000).

[7] I. A. Golutvin, V. V. Pal'chik, M. V. Savina, and S. V. Shmatov, Phys. At. Nucl. 70, 56 (2007).

[8] CMS Collaboration (S. Chatrchyan et al), JINST 3, S08004 (2008).

[9] CMS Collaboration (S. Chatrchyan et al). Phys. Rev. D 84, 112002 (2011).

[10] CMS Collaboration (V. Khachatryan et al), Eur. Phys. J. C 76, 325 (2016).

[11] J. C. Collins and D. E. Soper, Phys. Rev. D 16, 2219 (1977).

[12] S. Frixione, P. Nason, C. Oleari, JHEP 11, 07 (2007); S. Alioli, P. Nason, C. Oleari, E. Re, JHEP 07, 060 (2008); JHEP 06, 043 (2010).

[13] T. Sjostrand et al, Comp. Phys. Comm. 191, 159 (2015) 